\title{
Asymmetrical Eruption of Permanent Teeth in Australian Aborigines
}

\author{
P. Sobhi, S. Mihailidis*, J. R. Rogers, T. E. Hughes, G. C. Townsend \\ Craniofacial Biology Research Group, School of Dentistry, The University of Adelaide. Australia
}

ABSTRACT: A better understanding of the factors influencing tooth eruption is important given the association between altered eruption patterns and crowded or decayed teeth. Hence, the aims of this study were to quantify the extent of asymmetry in tooth eruption and to determine whether eruption asymmetry was significantly influenced by sex, tooth position or timing of emergence. Additionally, directionality of asymmetry and variation between ethnic groups were also explored. Data collection was based on the examination of serial dental casts from a sample of 90 Aborigines (50 male, 40 female) aged 6 to 18 years from the Yuendumu settlement in the Northern Territory of Australia. These casts were

The human dentition is a good model system for examining the nature and extent of asymmetries in morphology and development because teeth are arranged in the dental arches as antimeric pairs within different tooth classes, i.e., incisors, canines, premolars and molars. Dental asymmetry has been studied in relation to asymmetry type (fluctuating versus directional), dentition (primary or secondary), causes (genetic and environmental) and associated factors (sex, ethnicity and tooth or arch type). Previous studies of asymmetry in the human dentition have considered dental crown size, crown morphology and dental development, including tooth emergence and eruption (Garn and Bailey, 1977; Corruccini and Potter, 1981; Boklage, 1987; Kieser, 1990; Harris and Bodford, 2007).

Asymmetry is said to be directional when one side regularly displays greater and/or earlier development than the other. Directional asymmetry (DA) has been reported in the primary dentition (Townsend et al., 1999) while studies of the secondary dentition have shown both the presence (Lauterstein et al., 1967; Staley and Green, 1971; Harris, 1992) and absence (Townsend, 1985) of DA. Significant links between DA and emergence/ eruption timing have been described (Garn and Smith, 1980; Heikkinen et al., 1998) while more recent investigations have considered the relationship between DA and broader functional lateralities such as eyedness and handedness (Heikkinen et al., 2001). Random, nondirectional differences termed fluctuating asymmetry (FA) are thought to indicate an inability of the individual to buffer against developmental disturbances (Van Valen, obtained at yearly intervals from the 1950s to the early 1970s. Tooth antimeres on each cast were compared using a 4-grade eruption score. Relatively high (> 70\%) interand intra-observer concordances confirmed reliability of the recording system. Asymmetry frequencies were calculated and associations between variables assessed using chi-square analyses, with statistical significance set at alpha $=0.05$. Evidence of patterned asymmetry for permanent tooth eruption was noted among the sample of Australian Aborigines, with the distally positioned, laterforming teeth showing the highest levels of asymmetry. Dental Anthropology 2007;20:33-41.

1962). Hence, FA is considered to reflect the magnitude of developmental disturbances or, as Waddington (1957) termed, developmental noise. The effects of developmental noise or environmental stresses on dental asymmetry have been established using animal experiments with stressors such as noise, cold and heat (Bader, 1965; Siegel and Smookler, 1973; Siegel and Doyle, 1975; Siegel et al., 1977). Human studies, however, have measured the effects of stress using ethnicity, age and degree of modernization as proxies for stress. Studies have mostly cited greater asymmetry among indigenous (Kieser and Groenevald, 1988; Townsend, 1981), less contemporary (Black, 1980b) and older (Kieser et al., 1986) populations. Kieser and Groenevald (1988) proposed that it was not only the 'nature and severity of stress' but the 'ability of the individual to buffer against stress,' which may be useful in explaining results where either nil, or surprisingly significant associations between ethnicity and asymmetry (De Melo et al., 1975; Black, 1980b; Kieser et al., 1986; Kieser and Groenevald, 1988) have been found.

Local factors such as caries-infected primary teeth (Adler, 1963; Lauterstein et al., 1967), space constraints (Sofaer et al., 1971) and more general factors such as duration of odontogenesis (Townsend and Brown, 1980) have been used to explain why asymmetry is greater in the secondary dentition compared to the primary

${ }^{*}$ Correspondence to: Suzanna Mihailidis, School of Dentistry, University of Adelaide, Australia 5005 E-mail: suzanna.mihailidis@adelaide.edu.au 
dentition (Garn and Bailey, 1977). It is thought the more time spent in the pre-calcification stage may be linked to greater asymmetry between teeth (Mizoguchi, 1983, 1986) due to increased opportunity for environmental disturbances to deviate developing teeth away from their genetically determined paths.

While early studies by Garn et al. (1965) proposed the female double- $X$ chromosome provided greater buffering capacity and hence resulted in less asymmetry in females compared to males, more recent studies have not found significant variation by sex for either primary (Townsend, 1981; Townsend et al., 1999) or secondary (Lauterstein et al., 1967; Staley and Green, 1971) teeth. While some researchers have found no significant differences in dental asymmetry (cusp occurrence) between monozygotic, dizygotic and non-twins (Staley and Green, 1971), others have claimed that there are differences in dental asymmetry not only between different types of twins but also between twins and singletons (Boklage, 1987). Examples of geneticallylinked dental asymmetries, for example in Down Syndrome, have also been reported (Garn et al., 1970; Townsend, 1983). Studies reporting on arch-related asymmetry (Townsend, 1981; Kieser and Groenevald, 1988) have shown inconsistent findings while toothrelated asymmetry seems to strongly support the concept of morphogenetic fields proposed by Butler (1939) and subsequently modified by Dahlberg (1945). This pattern of asymmetry is well cited in the literature (Garn and Bailey, 1977; Townsend, 1981) with few exceptions (Kieser and Groenevald, 1988).

In regards to tooth form, numerous studies can be found concerning antimeric variation in tooth size and shape (Garn and Bailey, 1977; Black 1980a,b; Harris and Nweeia, 1980; Townsend, 1981, 1985; Kieser et al., 1986; Kieser and Groenevald, 1988; Townsend et al., 1999; Khalaf et al., 2005) but relatively fewer investigations that have considered asymmetry in the timing of tooth eruption among human populations (Tomes, 1859; Lysell et al., 1962; Lauterstein et al., 1967; Staley and Green, 1971; De Melo et al., 1975; Nystrom, 1977; Garn and Smith, 1980; Heikkinen et al., 1998, 2001). Tomes (1859) suggested that teeth on the left side erupt earlier than those on the right while Lysell et al. (1962) also reported similar findings in their study of primary tooth emergence in Swedish children. Still, there are studies that have failed to detect any significant differences in timing of emergence between antimeric pairs (Staley and Green, 1971; De Melo et al., 1975; Nystrom, 1977). More recent studies by Heikkinen et al. (1998) have established significant associations of sex and ethnicity on timing of tooth eruption by comparing samples of Finnish and US children. An exciting recent development has been the link made between eruption asymmetries and functional lateralities by examining tooth eruption sequence as an indicator in the timing of overall laterality (Heikkinen et al., 2001).

While information regarding the existence of systematic relationships or patterned asymmetry is growing, there are still many aspects of odontogenic mechanisms-one of which includes dental eruption asymmetry - that are yet to be fully understood.

Hence the aims of this study were to describe the nature and extent of asymmetry in permanent tooth eruption among a sample of Australian Aborigines.

Several specific hypotheses were considered in relation to tooth eruption:

1. More distally placed teeth within each tooth class will display greater asymmetry than more mesially placed teeth (Butler's field theory)

2. Females will exhibit greater symmetry than males (better buffering with two X chromosomes)

3. Later-erupting teeth will have higher levels of asymmetry compared to earlier-erupting teeth (longer time spent in development increases exposure to environmental disturbances)

4. Directional asymmetry in tooth eruption will exist for some teeth (some evidence from previous studies)

5. Variation in tooth eruption symmetry will exist between Australian Aborigines and other ethnic groups (previous findings in other ethnic groups)

\section{STUDY POPULATION AND METHODS}

This study was based on the examination of serial dental casts obtained at approximately yearly intervals of 90 Australian Aborigines, 50 males and 40 females, stored in the Murray Barrett Laboratory of the Adelaide Dental Hospital. The casts had been prepared during visits of anthropological research teams from the University of Adelaide to the Yuendumu settlement from the 1950s until the early 1970s. Yuendumu settlement, situated approximately $300 \mathrm{~km}$ northwest of Alice Springs in the Northern Territory of Australia, was created in 1946 and comprised a relatively selfcontained Aboriginal population. The people belonged predominantly to the Wailbri and Pintubi tribes and were of pure Aboriginal ancestry as far as could be ascertained. From a cultural point of view, the group was still in a transition stage-from a food gathering/ hunting society to a reliance on Western civilization for its basic needs. The geographically isolated conditions at Yuendumu provided a rare opportunity to study a group of people who had not yet been influenced to any great extent by the effects of European culture.

Damaged casts and those displaying missing teeth were excluded from the sample. Every effort was made to include individuals with the maximum number of available serial dental casts during the period of eruption of the permanent teeth. The ages of the subjects were between 6 and 18 years. The method of scoring tooth eruption followed that of Heikkinen et al. (1999) where tooth antimeres on each cast were compared 
using a 4-grade eruption score. Each of the eight pairs of permanent teeth in both the maxilla and mandible were scored for eruption status. The eruption stages were defined as follows:

1. Tooth not emerged

2. Occlusal surface of the tooth recently emerged

3. Tooth crown half erupted

4. Eruption of tooth complete or nearly complete

The term "emergence" is used to refer to the point at which a tooth appears in the oral cavity, and the term "eruption" refers to the process by which a tooth moves into occlusion with its opponent(s). The cast on which a tooth was first evident was used for scoring the stage of eruption of that particular tooth. Teeth were given a score of 1 to 4 according to their eruption status. Most of the tooth pairs that were included in the analysis were in the erupting phase; that is, they were scored as 2 or 3 , but a small number of tooth pairs where one tooth was scored as a 1 and the antimere as a 4 , were also included. Tooth pairs were not included if they were both scored as 1 or 4 . Inter- and intra-observer reliability in scoring was determined from double determinations on 20 randomly selected casts. Where discrepancies occurred, the casts were re-examined, and a decision was made as to the appropriate score.

Chi-square tests were used to test for significant variation in tooth eruption status between:

- teeth within a tooth class, namely I1 versus I2, P1 versus P2, M1 versus M2;

- Australian Aboriginal male and female children; and

- Australian Aboriginal, American and Finnish children (where data reported by Heikkinen et al. (1999) were used for making comparisons).

Statistical analysis was conducted using SPSS software with significance set at the conventional level of alpha $=0.05$.

\section{RESULTS}

Table 1 presents the inter-observer and intraobserver percentage concordances for scoring eruption status based on double determinations. Generally, the concordance percentages were higher in the mandible compared with those in the maxilla. For example, concordance for scoring eruption of the lower laterals

TABLE 1. Inter-and intra-observer percentage concordances for eruption status ${ }^{1}$

\begin{tabular}{crrrr}
\hline Tooth number & \multicolumn{2}{c}{$\begin{array}{c}\text { Inter-observer } \\
(\% \text { concordance) }\end{array}$} & \multicolumn{2}{c}{$\begin{array}{c}\text { Intra-observer } \\
\text { (\% concordance })^{2}\end{array}$} \\
\hline 11,21 & 85 & 85 & 90 & 95 \\
12,22 & 85 & 75 & 70 & 85 \\
13,23 & 95 & 80 & 100 & 90 \\
14,24 & 95 & 95 & 100 & 100 \\
15,25 & 75 & 90 & 85 & 90 \\
16,26 & 85 & 80 & 95 & 95 \\
17,27 & 90 & 85 & 95 & 95 \\
18,28 & 85 & 90 & 95 & 95 \\
31,41 & 90 & 90 & 95 & 95 \\
32,42 & 100 & 100 & 100 & 100 \\
33,43 & 95 & 95 & 95 & 95 \\
34,44 & 75 & 75 & 80 & 90 \\
35,45 & 95 & 95 & 100 & 95 \\
36,46 & 85 & 85 & 90 & 90 \\
37,47 & 80 & 90 & 95 & 100 \\
38,48 & 80 & 90 & 85 & 100 \\
\hline
\end{tabular}

${ }^{1}$ Notes:

a) Sample size for comparisons $=20$ subjects

b) Number of individual teeth $=17$ to 20

c) The notation system used for tooth identification in Tables 1-5 is the Federation Dentaire Internationale (FDI) notation that is based on a two-digit system. The first digit identifies the quadrant (numbered 1-4 for permanent teeth, beginning at the patient's upper right and proceeding in a clockwise direction) while the second digit identifies the tooth within the quadrant (numbered 1-8 and beginning from the midline) e.g., a maxillary right permanent central incisor is a 11 and a mandibular left permanent third molar is a 38.

2 The two numbers refer to percent concordance scored on each of the two teeth (homologous teeth on left and right quadrants). For example, there was $85 \%$ inter-observer concordance for the right (tooth 11) and left (tooth 21) maxillary central incisor, whereas intra-observer concordance was 90\% and 95\%, respectively for teeth 11 and 21. 
TABLE 2. Symmetrical-asymmetrical eruption by tooth class

\begin{tabular}{|c|c|c|c|c|c|c|}
\hline \multirow[b]{2}{*}{ Tooth class } & \multirow{2}{*}{$\begin{array}{l}\text { Tooth } \\
\text { number }\end{array}$} & \multirow{2}{*}{$\begin{array}{c}\text { Teeth in } \\
\text { active phase } \\
n\end{array}$} & \multicolumn{2}{|c|}{$\begin{array}{l}\text { Symmetrical } \\
\text { eruption }\end{array}$} & \multicolumn{2}{|c|}{$\begin{array}{l}\text { Asymmetrical } \\
\text { eruption }\end{array}$} \\
\hline & & & $\mathrm{n}$ & $\%$ & $\mathrm{n}$ & $\%$ \\
\hline \multirow{2}{*}{ Incisor } & $11,21,31,41$ & 98 & 78 & 80 & 20 & 20 \\
\hline & $12,22,32,42$ & 132 & 91 & 69 & 41 & 31 \\
\hline \multirow[t]{2}{*}{ Premolar } & $14,24,34,44$ & 109 & 45 & 41 & 64 & 59 \\
\hline & $15,25,35,45$ & 99 & 30 & 30 & 69 & 70 \\
\hline \multirow[t]{3}{*}{ Molar * } & $16,26,36,46$ & 74 & 49 & 66 & 25 & 34 \\
\hline & $17,27,37,47$ & 138 & 76 & 55 & 62 & 45 \\
\hline & $18,28,38,48$ & 98 & 40 & 41 & 58 & 59 \\
\hline
\end{tabular}

${ }^{*}$ chi-square analysis $(\mathrm{P}<0.05)$

was $100 \%$ for both sets of observations. Generally, there was higher concordance for intra-observer comparisons than for inter-observer comparisons but, overall, the percentage concordances were relatively high indicating that the scoring system was reliable and that errors in scoring were unlikely to bias the results.

Table 2 presents the percentages of teeth showing symmetrical and asymmetrical eruption in the Australian
Aboriginal sample with results categorized by tooth class. Initial analyses did not uncover significant variation by sex and therefore data were combined to increase sample sizes. The results indicate significantly higher asymmetry for the more distal teeth in the molar tooth class supporting the theory of morphogenetic fields (Dahlberg, 1945). For example, less than half $(41 \%)$ of third molars exhibited symmetrical eruption

TABLE 3. Symmetrical eruption by sex and emergence phase ${ }^{1}$

\begin{tabular}{|c|c|c|c|c|c|c|}
\hline \multirow{2}{*}{$\begin{array}{l}\text { Tooth } \\
\text { number }\end{array}$} & \multirow{2}{*}{$\begin{array}{c}\text { Mean emergence } \\
\text { males } \\
\text { (years) }\end{array}$} & \multirow{2}{*}{$\begin{array}{c}\text { Mean emergence } \\
\text { females } \\
\text { (years) }\end{array}$} & \multicolumn{2}{|c|}{$\begin{array}{l}\text { Symmetrical } \\
\text { eruption, males }\end{array}$} & \multicolumn{2}{|c|}{$\begin{array}{c}\text { Symmetrical } \\
\text { eruption, females }\end{array}$} \\
\hline & & & $\mathrm{n}$ & $\%$ & n & $\%$ \\
\hline \multicolumn{7}{|c|}{ First Emergence Phase ${ }^{2}$} \\
\hline 36,46 & 6.4 & 5.1 & 8 & 44 & 5 & 83 \\
\hline 16,26 & 6.4 & 5.7 & 22 & 81 & 14 & 82 \\
\hline 31,41 & 6.6 & 6.4 & 15 & 71 & 11 & 79 \\
\hline 11,21 & 7.0 & 7.3 & 32 & 82 & 20 & 83 \\
\hline 32,42 & 7.2 & 7.3 & 29 & 76 & 18 & 72 \\
\hline 12,22 & 8.5 & 8.1 & 21 & 57 & 23 & 72 \\
\hline \multicolumn{7}{|c|}{ Second Emergence Phase } \\
\hline 33,43 & 10.0 & 9.1 & 20 & 49 & 21 & 62 \\
\hline 14,24 & 10.3 & 9.8 & 13 & 41 & 15 & 48 \\
\hline 34,44 & 10.5 & 9.9 & 9 & 36 & 8 & 38 \\
\hline 13,23 & 10.5 & 10.1 & 24 & 59 & 13 & 38 \\
\hline 37,47 & 11.2 & 10.8 & 16 & 46 & 17 & 63 \\
\hline 15,25 & 11.4 & 11.0 & 8 & 30 & 9 & 38 \\
\hline 35,45 & 11.5 & 11.0 & 8 & 28 & 5 & 26 \\
\hline 17,27 & 11.5 & 11.0 & 25 & 61 & 18 & 51 \\
\hline 18,28 & 16.8 & 16.1 & 9 & 38 & 8 & 36 \\
\hline 38,48 & 16.5 & 16.1 & 11 & 37 & 12 & 55 \\
\hline
\end{tabular}

${ }^{1}$ No significant association between sex and symmetrical eruption $(\mathrm{P}>0.05)$ but significant assocation between emergence phase and symmetrical eruption status $(\mathrm{P}<0.05)$ based on chi-square analyses.

${ }^{2}$ First emergence phase refers to the time interval during which the tooth groups 36, 46 to 12, 22 emerge, between 5.1 and 8.5 years. Second emergence phase refers to the time interval during which the tooth groups 33,43 to 38,48 emerge between 9.1 and 16.8 years of age.

Reference: Mean emergence times (Brown et al., 1979) 
TABLE 4. Distribution of directionality among asymmetrically erupting antimeres

\begin{tabular}{|c|c|c|c|c|c|}
\hline \multirow{2}{*}{$\begin{array}{l}\text { Tooth } \\
\text { number }\end{array}$} & \multirow{2}{*}{$\begin{array}{c}\text { Teeth in } \\
\text { active phase } \\
\mathrm{n}\end{array}$} & \multicolumn{2}{|c|}{$\begin{array}{l}\text { Asymmetrical } \\
\text { eruption } \\
R>L\end{array}$} & \multicolumn{2}{|c|}{$\begin{array}{c}\text { Asymmetrical } \\
\text { eruption } \\
L>R\end{array}$} \\
\hline & & $\mathrm{n}$ & $\%$ & $\mathrm{n}$ & $\%$ \\
\hline 11,21 & 11 & 7 & 64 & 4 & 36 \\
\hline 12,22 & 25 & 16 & 64 & 9 & 36 \\
\hline 13,23 & 38 & 21 & 55 & 17 & 45 \\
\hline 14,24 & 35 & 17 & 49 & 18 & 51 \\
\hline 15,25 & 34 & 18 & 53 & 16 & 47 \\
\hline 16,26 & 8 & 4 & 50 & 4 & 50 \\
\hline 17,27 * & 33 & 5 & 15 & 28 & 85 \\
\hline 18,28 & 29 & 11 & 38 & 18 & 62 \\
\hline 31,41 & 9 & 5 & 56 & 4 & 44 \\
\hline 32,42 * & 16 & 12 & 75 & 4 & 25 \\
\hline 33,43 * & 34 & 23 & 68 & 11 & 32 \\
\hline 34,44 * & 29 & 20 & 69 & 9 & 31 \\
\hline 35,45 * & 35 & 25 & 71 & 10 & 29 \\
\hline 36,46 * & 17 & 2 & 12 & 15 & 88 \\
\hline 37,47 & 29 & 14 & 48 & 15 & 52 \\
\hline 38,48 & 29 & 15 & 52 & 14 & 48 \\
\hline
\end{tabular}

${ }^{*}$ chi-square analysis $(\mathrm{P}<0.05)$

compared to two thirds (66\%) of first molars.

Results comparing the percentage of antimeric pairs showing symmetrical eruption against mean emergence times for Australian Aboriginal males and females (Brown et al., 1979) are presented in Table 3 . While no significant association was found between symmetrical eruption frequencies and sex, there was a significant association between phase of emergence and symmetrical eruption, with later-erupting teeth (represented in the second emergence phase) displaying significantly higher levels of asymmetrical eruption than the earlier-erupting teeth (represented in the first phase of emergence).

Table 4 provides percentages of teeth showing directional eruption asymmetry in Australian Aborigines. Initial analyses did not uncover significant variation by sex and therefore data were combined to

TABLE 5. Symmetrical eruption by ethnic group

\begin{tabular}{|c|c|c|c|c|c|c|c|c|}
\hline \multirow{2}{*}{$\begin{array}{l}\text { Tooth } \\
\text { number }\end{array}$} & \multicolumn{2}{|c|}{$\begin{array}{c}\text { US } \\
\text { Caucasian }\end{array}$} & \multicolumn{2}{|c|}{$\begin{array}{c}\text { US } \\
\text { African American }\end{array}$} & \multicolumn{2}{|c|}{ Finnish } & \multicolumn{2}{|c|}{$\begin{array}{l}\text { Australian } \\
\text { Aboriginal }\end{array}$} \\
\hline & $\mathrm{n}$ & $\%$ & $\mathrm{n}$ & $\%$ & $\mathrm{n}$ & $\%$ & $\mathrm{n}$ & $\%$ \\
\hline 11,21 * & 123 & 51 & 117 & 58 & 262 & 86 & 52 & 83 \\
\hline 12,22 * & 87 & 52 & 116 & 46 & 222 & 74 & 44 & 64 \\
\hline 13,23 * & 10 & 53 & 45 & 42 & 202 & 66 & 37 & 49 \\
\hline 14,24 * & 6 & 16 & 39 & 28 & 175 & 58 & 28 & 44 \\
\hline 15,25 * & 2 & 7 & 4 & 5 & 158 & 54 & 17 & 33 \\
\hline 16,26 & 276 & 78 & 359 & 83 & 253 & 83 & 36 & 82 \\
\hline 17,27 & 11 & 61 & 123 & 72 & 205 & 68 & 43 & 57 \\
\hline 31,41 * & 481 & 93 & 333 & 98 & 270 & 89 & 26 & 74 \\
\hline 32,42 * & 145 & 53 & 154 & 63 & 234 & 77 & 47 & 75 \\
\hline 33,43 * & 9 & 29 & 124 & 57 & 195 & 64 & 41 & 55 \\
\hline 34,44 * & 17 & 45 & 32 & 24 & 183 & 60 & 17 & 37 \\
\hline 35,45 * & 2 & 13 & 4 & 5 & 180 & 62 & 13 & 27 \\
\hline 36,46 * & 142 & 66 & 133 & 72 & 256 & 84 & 13 & 43 \\
\hline 37,47 & 10 & 63 & 64 & 57 & 202 & 67 & 33 & 53 \\
\hline
\end{tabular}

*chi-square analysis $(\mathrm{P}<0.05)$ 
increase sample sizes. Significantly advanced rightsided eruption was found for the mandibular lateral incisors, canines and first and second premolars, while significantly advanced left-sided eruption was evident for the maxillary second molars and lower first molars.

Proportional estimates of symmetrically erupting antimeric tooth pairs from a cross-sectional sample of 2092 African American and Caucasian American children and a longitudinal sample of 481 Finnish children (Heikkinen et al., 1999) are presented alongside estimates obtained for Australian Aborigines in the current study (Table 5). Chi-square tests comparing the extent of asymmetry between the four samples yielded statistically significant results, with all teeth except the maxillary first and second molars and mandibular second molars exhibiting significant variation by ethnic group. There were, however, limitations in comparing between these studies, including differences in sample sizes and sex distribution within the samples. Although the same recording system was used, inter-observer comparisons could not be carried out to determine reliability. Hence, significant findings should be considered with caution given the limitations in making comparisons between studies. Overall trends included generally higher levels of eruption symmetry among the Finnish and Australian Aboriginal samples compared to the US African American and Caucasian samples. Antimeric tooth pairs that were found to exhibit the greatest level of stability across ethnic groups included the early emerging lower central incisors and upper first molars. In almost all cases, the more distal teeth in each morphogenetic field tended to be more asymmetrical across all samples. There were, however, exceptions: upper lateral incisors (US Caucasian sample), lower second premolars (Finnish sample) and lower lateral incisors and second molars (Australian Aboriginal sample).

\section{DISCUSSION}

This study adds to the availableinformation regarding patterns of asymmetry observed for permanent tooth eruption, which relates to: morphogenetic fields, increased buffering capacity of females, timing of emergence, and directional asymmetry. The influence of ethnicity, as an indicator of environmental stress influencing eruption symmetry, was less clear. There were obvious limitations when comparing samples from different studies related to differences in methodological approaches. Despite these limitations, some apparent general trends were evident.

The results pertaining to our first hypothesis (Table 2) are in support of Butler's field theory (Butler, 1939; Dahlberg, 1945), based on the concept that distal teeth within each morphogenetic class tend to be more variable than the more mesially positioned key tooth. This tends to occur with the exception of the lower lateral and central incisors, which exhibit the opposite pattern and are often considered to be an exception to the rule. These findings are also well in accordance with the general literature (Garn and Bailey, 1977; Townsend, 1981; Kieser et al., 1986; Khalaf et al., 2005). The second hypothesis was not supported in that no significant difference was found to exist between males and females (Table 3), while later-emerging teeth did exhibit significantly greater asymmetry (Table 3 ) lending support to the third hypothesis related to timing of tooth emergence and degree of eruption asymmetry. Heikkinen's study (1998) derived similar findings with regards to the proportion of symmetrically erupting antimeric pairs, which was found to be approximately $90 \%$ in mandibular central incisors, while the maxillary lateral incisors, which are the last teeth to emerge in the first phase of the mixed dentition, showed a symmetrical proportion of approximately 50\%. With later-emerging teeth, the proportion of symmetrically erupting antimeres decreased, being the lowest in second premolars. Interestingly, the last emerging permanent teeth in the second phase of the mixed dentition, the second molars, appeared to be more symmetric. Given that the more distal teeth in each class tend to emerge later in life and spend relatively more time erupting, it is plausible that asymmetry will be greater due to the larger window of opportunity for environmental influences to interfere with the eruption patterns of these teeth. Recent research by Parner et al. (2002) has reported on the apparent correlation between emergence times of teeth belonging to the same jaw innervation group. The authors also suggested that maxillary teeth may exhibit greater stability as their nerve innervation is more distinct for individual teeth. These findings might then be extended in relation to dental asymmetry patterns. For example, arch-specific findings related to dental asymmetry have shown the maxillary arch to exhibit greater asymmetry compared to the mandibular arch (Townsend, 1981; Kieser et al., 1986).

Broader links between prenatal factors and dental eruption patterns have also been identified (Garn et al., 1965; Heikkinen et al., 1998). Heikkinen et al. (1995) found the early clinical eruption of permanent central incisors was slightly affected in children born to mothers who smoked during pregnancy, while Garn et al. (1965) explained the large differences found between monozygotic twins as being possibly due to the effects of steroids on root formation and tooth movement. More research investigating the broader links between dental asymmetry patterns and other developmental disturbances should be considered for the future.

Garn et al. (1967) studied dental crown dimensions in males and females and proposed that increased dental asymmetry observed in males may be related to the presence of only one $X$-chromosome. Females with a pair of X-chromosomes were found to be more symmetrical in tooth size and it was proposed this may be related to 
the extra X-chromosome leading to increased buffering capacity. As discussed earlier, several studies following Garn et al. (1967) do not report significant sex variation. No significant difference between males and females was noted in our study, while more recent studies by Heikkinen et al. (1998) do show significant difference by sex with females being more symmetric than males. Heikkinen found this pattern for the maxillary lateral incisor and first premolar, and the mandibular central incisor and canine in US children, and for the maxillary first premolar and mandibular central incisor and first molar in Finnish children. Similar findings with respect to greater symmetry among females have been documented by Bailit et al. (1970) and Townsend and Brown (1980), lending further support to Garn's theory. Overall, there appears to be inconsistency in regards to the influence of sex on asymmetry patterns.

Our fourth hypothesis regarding directionality in tooth eruption was also supported with results indicating left-sided dominance for the maxillary second and third molars and mandibular first molar, and right-sided dominance for the maxillary incisors and mandibular lateral incisors, canines and premolars. While our findings do indicate the existence of directionality, the underlying pattern is less clear. Harris (1992) and Townsend et al. (1999) suggested left-sided dominance in tooth size in one arch may be associated with rightsided dominance in the opposite arch however this did not seem evident in our sample.

For testing the last hypothesis, comparisons were drawn between data from the present study and those from Heikkinen et al. (1998) for US and Finnish children. Overall trends included generally greater eruption symmetry among the Finnish and Australian Aboriginal samples compared to the US African American and Caucasian samples. Antimeric tooth pairs that were found to exhibit the greatest level of stability across ethnic groups included the early emerging lower central incisors and upper first molars. In almost all cases, the more distal teeth in each morphogenetic field tended to be more asymmetrical across all samples. It is plausible that the upper first molars would show similar trends across samples given that these teeth are important in stabilizing occlusion and are also less likely to be interrupted in their eruption path as they do not succeed primary teeth. However, other results are more surprising. For example, the lower central incisors tend to be more variable than lower lateral incisors in one sample (Australian Aborigines) but not so for the other three samples. Similarly, it is not immediately obvious why the Finns and Australian Aborigines would be more similar in their patterns of symmetry compared with the American samples.

A confounding factor in making these comparisons is that each of the three samples was scored by a different researcher. Whilst the same scoring system was used in each case, it is possible that inconsistencies exist between scorers. Hence, considerable caution should be exercised when making inter-population comparisons of existing data on eruption asymmetry due to these limitations. Further studies based on larger sample sizes and consistent methodology across samples are required before true inter-population variation in eruption asymmetry can be described accurately.

Overall methodological considerations should also be taken into account. The number of subjects included in this study was limited to 90 from a total sample of 450 individuals. Many individuals were excluded because of limited numbers of serial casts being available during the mixed dentition phase, and casts for some individuals could not be included because they were damaged or had missing teeth. Teeth were scored using the cast on which they first became evident, although subsequent casts, that were usually obtained at annual intervals, were used to confirm presence of teeth and assist in scoring. While limitations of the scoring system were noted, accurate assessment of the degree of eruption was not always possible. There were several teeth that displayed gingival recession or inflamed gingivae, which in turn led to more challenging judgement of whether eruption was full or partial. In some instances, examination of the level of the occlusal plane and physically occluding the upper and lower casts was necessary in order to determine whether a tooth was fully or partially erupted. There were problems in some cases in distinguishing between categories 2 and 3, and categories 3 and 4 . It would be worthwhile considering the addition of an extra score(s) to define more precise points of eruption, although this may reduce the level of reliability of scoring.

\section{CONCLUSION}

Our findings provide evidence of patterned tooth eruption asymmetry among a sample of Australian Aborigines with the distally positioned, later-forming teeth showing the highest levels of asymmetry. While directional asymmetry was shown to be present, no clear pattern could be ascertained. Despite limitations in making comparisons across different studies, there was some evidence to suggest stability of the upper first molars and the lower central incisors when investigating the influence of ethnicity on asymmetry patterns.

\section{ACKNOWLEDGEMENTS}

The dental casts were collected by the late M. J. Barrett during a growth study which was supported by Research Grant DE 02034 from the National Institute of Dental Research, Bethesda, MD, and by grants from The University of Adelaide and the Australian Institute of Aboriginal Studies, Canberra. Ethical approval was obtained at the time of data collection for the study. 


\section{LITERATURE CITED}

Adler P. 1963. Effect of some environmental factors on sequence of permanent tooth eruption. J Dent Res 42:605-16.

Bader RS. 1965. Fluctuating asymmetry in the dentition of the house mouse. Growth 29:291-300.

Bailit HL, Workmen PL, Niswander JD, MacLean CJ. 1970. Dental asymmetry as an indicator of genetic and environmental conditions in human populations. Hum Biol 42:626-638.

Black TK. 1980a. Fluctuating asymmetry in the deciduous dentition. J Dent Res 59:725.

Black TK. 1980b. An exception to the apparent relationship between stress and fluctuating dental asymmetry. J Dent Res 59:1168-1169.

Boklage CE. 1987. Developmental differences between singletons and twins in distributions of dental diameter asymmetries. Am J Phys Anthropol 74:319-331.

Brown T, Jenner JD, Barrett MJ, Lees GH. 1979. Exfoliation of deciduous teeth and gingival emergence of permanent teeth in Australian Aborigines. Canberra: Australian Institute of Aboriginal Studies. Occasional Papers in Human Biology 1:47-70.

Butler PM. 1939. Studies of the mammalian dentition. Differentiation of the post-canine dentition. Proc Zool Soc London 109:1-36.

Corruccini RS, Potter RH. 1981. Developmental correlates of crown component asymmetry and occlusal discrepancy. Am J Phys Anthropol 55:21-31.

Dahlberg AA. 1945. The changing dentition of man. J Am Dent Assoc 32:676-690.

De Melo MJ, Freitas E, Salzano FM. 1975. Eruption of permanent teeth in Brazilian Whites and Blacks. Am J Phys Anthropol 42:145-150.

Garn SM, Lewis AB, Kerewsky RS. 1965. Genetic, nutritional and maturational correlates of dental development. J Dent Res 44:228-242.

Garn SM, Cohen MM, Geciauskas MA. 1970. Increased crown-size asymmetry in trisomy G. J Dent Res 49:465.

Garn SM, Bailey SM. 1977. The symmetrical nature of bilateral asymmetry of deciduous and permanent teeth. J Dent Res 56:1422.

Garn SM, Lewis AB, Kerewsky RS. 1967. Buccolingual size asymmetry and its developmental meaning. Angle Orthod 37:186-193.

Garn SM, Smith BH. 1980. Patterned asymmetry in tooth emergence timing. J Dent Res 59:1526-1527.

Harris EF. 1992. Laterality in human odontometrics: analysis of a contemporary American White series. In: Lukacs JR, editor. Culture, ecology and dental anthropology. Delhi: Kamla-Raj, p 157-170.

Harris EF, Bodford K. 2007. Bilateral asymmetry in the tooth relationships of orthodontic patients. Angle Orthod 77:779-786.
Harris EF, Nweeia MT. 1980. Dental asymmetry as a measure of environmental stress in the Ticuna Indians of Colombia. Am J Phys Anthropol 53:133-142.

Heikkinen T, Alvesalo L, Osborne RH, Tierni J, Virtanen J. 1999. The effect of sex and race on the symmetry of the permanent tooth clinical eruption. In: Mayhall JT, Heikkinen T, editors. Dental morphology 1998. Oulu: Oulu University Press, p 473-482.

Heikkinen T, Alvesalo L, Osborne RH, Tienari J. 2001. Tooth eruption symmetry in functional lateralities. Arch Oral Biol 46:609-617.

Kieser JA. 1990. Fluctuating odontometric asymmetry. In: Lasker GW, Mascie-Taylor CGN, Roberts DF, editors. Human adult odontometrics-the study of variation in adult tooth size. Cambridge: Cambridge University Press. pp 96-104.

Kieser JA, Groenevald HT. 1988. Fluctuating odontometric asymmetry in an urban South African black population. J Dent Res 67:1200-1205.

Kieser JA, Groenevald HT, Preston CB. 1986. Fluctuating odontometric asymmetry in the Lengua Indians of Paraguay. Ann Hum Biol 13:489-498.

Khalaf K, Elcock C, Smith RN, Brook AH. 2005. Fluctuating dental asymmetry of multiple crown variables measured by an image analysis system. Arch Oral Biol 50:249-253.

Lauterstein AM, Pruzansky S, Levine NL. 1967. Bilateral asymmetry in mandibular tooth development. J Dent Res 46:279-285.

Lysell L, Magnusson B, Thilander B. 1962. Time and order of eruption of the primary teeth. Odont Rev 13:217-234.

Mizoguchi Y. 1983. Factor analysis of environmental variation in the permanent dentition. Bull Natl Sci (Tokyo) 6:29-46.

Mizoguchi Y. 1986. Correlated asymmetries detected in the tooth crown diameters of human permanent teeth. Bull Natl Sci (Tokyo) 12:24-45.

Nystrom M. 1977. Clinical eruption of deciduous teeth in a series of Finnish children. Proc Finn Dent Soc 73:155-161.

Parner ET, Heidmann JM, Kjaer I, Vaeth M, Poulsen S. 2002. Biological interpretation of the correlation of emergence times of permanent teeth. J Dent Res 81:451-454.

Siegel MI, Doyle WJ. 1975. The effects of cold stress on fluctuating asymmetry in the dentition of the mouse. J Exp Zool 193:385-389.

Siegel MI, Smookler HH. 1973. Fluctuating dental asymmetry and audiogenic stress. Growth 37:35-39.

Siegel MI, Doyle WJ, Kelley C. 1977. Heat stress, fluctuating asymmetry and prenatal selection in the laboratory rat. Am J Phys Anthropol 46:121-126.

Sofaer JA, Chung CS, Niswander JD, Runck DW. 1971. Developmental interaction, size, and agenesis among permanent maxillary incisors. Hum Biol 43:36-45. 
Staley RN, Green LJ. 1971. Bilateral asymmetry in tooth cusp occurrence in human monozygotic twins, dizygotic twins, and nontwins. J Dent Res 50:83-89.

Tomes J. 1859. A system of dental surgery, 2nd ed. Philadelphia: Lindsay and Blakiston.

Townsend GC. 1981. Fluctuating asymmetry in the deciduous dentition of Australian Aboriginals. J Dent Res 60:1849-1857.

Townsend GC. 1983. Fluctuating dental asymmetry in Down's syndrome. Aust Dent J 28:39-44.

Townsend GC. 1985. Intercuspal distances of maxillary pre-molar teeth in Australian Aboriginals. J Dent Res 64:443-446.
Townsend GC, Brown T. 1980. Dental asymmetry in Australian Aboriginals. Hum Biol 52:661-673.

Townsend GC, Dempsey P, Richards LC. 1999. Asymmetry in the deciduous dentition: fluctuating and directional components. Perspectives in Human Biology 4:45-52.

Van Valen L. 1962. A study of fluctuating asymmetry. Evolution 16:125-142.

Waddington CH. 1957. The strategy of the genes. London: George Allen and Unwin. 